\title{
EDITORIAL
}

\section{Otra vuelta de tuerca al sector de la salud}

Tal como se comentó en la edición anterior de esta Revista, el sistema Isapre ha sufrido una pérdida sustancial de beneficiarios desde 1998. En efecto, en 8 años han decrecido en un 32\%, disminución que se explica principalmente por desafiliaciones voluntarias $(65 \%)$, concentradas en cotizantes de más bajo poder adquisitivo, pero también de menor edad y número de cargas. Esta situación se ha prolongado mucho más allá de la crisis económica que explicó esta tendencia a comienzos de la década.

En paralelo, los mejores resultados económicos de las Isapre -y también su creciente concentraciónexplican la actitud pasiva de las mismas frente al fenómeno de desafiliación que las aqueja. En buenas cuentas, las Instituciones de Salud Previsional han perdido importancia dentro del sistema de seguridad social en salud en Chile y Fonasa -el seguro público- la ha recuperado. Hoy empieza a ser más conveniente para algunas personas "mezclar" la cobertura básica que ofrece Fonasa con un seguro complementario ofrecido por compañías privadas, con la ventaja en flexibilidad que implica poder mejorar las coberturas en aquellos ámbitos más justificados o preferidos, como por ejemplo la atención hospitalaria o los eventos catastróficos, y menos en lo ambulatorio. Esto significa que la amenaza de desafiliación de las Isapre no ha terminado aún.

A lo anterior cabe agregar un giro en la mirada del Gobierno hacia la salud pública -como disciplina o enfoque-, y también una renovada confianza en el sistema público prestador de servicios de salud. Destaca en el programa del Ministerio una evidente preocupación por los niños y el adulto mayor, por la salud mental y reproductiva y por los estilos de vida saludables. En lo referente a la organización y al financiamiento de la prestación de servicios médicos, la continuidad del Auge parece ser el eje principal, al que se agrega el interés por el mejoramiento de la salud previsional privada y el fortalecimiento de la Atención Primaria, basados en un modelo de centros de salud pequeños, de administración puramente municipal. En el Parlamento, como era previsible, el proyecto de ley de Derechos y Deberes de los Pacientes, que había quedado pendiente desde la gestión de la entonces ministra de salud y hoy Presidenta, Michelle Bachelet, ha sido retomado para su discusión.

La idea de modificar la naturaleza de las Isapre para hacerlas socialmente aceptables y arraigarlas en los cimientos de la seguridad social -el otrora denominado "fondo solidario" del que se las quería hacer partícipes- parece definitivamente descartada. Los costos de esa transformación eran muy elevados, ya que incluían la idea de portabilidad de los subsidios, el uso de las camas de los hospitales públicos, el riesgo en la política fiscal de una duplicación de gastos, etc. Al parecer, se transita hacia un modelo de seguridad social en salud con un solo asegurador y probablemente también -al ritmo que se considere prudente imprimirle- hacia una mayor apertura de la prestación de servicios por prestadores privados a beneficiarios del sistema público, como producto del Auge.

El Auge en sí mismo continúa siendo un desafío de envergadura. Ya hay quienes aseguran que en la práctica las garantías han sido modificadas por aumento de precios en las prestaciones y aumento en los tiempos de espera. A esto, que está en plena discusión, se agregaría un hipotético pero previsible desplazamiento de las prestaciones no Auge en los hospitales, el que debería incrementarse en la medida en que se agreguen nuevas prestaciones a la garantía. Esto perfila dónde estará el foco del debate sectorial en los próximos meses; en tanto, el marco de transparencia que la Presidenta quiere imprimirle a su Gobierno obligará a un gran esfuerzo de demostración al sector, sobre el cual recaerá el "peso de la prueba", ahora con garantías explícitas cuyo cumplimiento o incumplimiento puede ser objetivado.

Volviendo a la salud pública, la creación de la Comisión Presidencial Asesora para Políticas de la Infancia, permite aventurar un futuro positivo para un tema prácticamente olvidado en la 
inercia del histórico programa de control de niño sano, cuya renovación es necesaria. Del mismo modo, los avances hacia la transformación del modelo de atención de los problemas de salud mental nos parecen también promisorios. Así también, aunque comunicacionalmente se pudiese haber adelantado a su momento en la agenda del Gobierno, la señal dada en torno a la salud reproductiva también nos ha parecido muy saludable.

Las señales que leemos desde la Revista Chilena de Salud Pública sólo pueden ser interpretadas como una oportunidad para que el saber que está siendo originado en el mundo académico y puesto en conocimiento de la comunidad, pueda ser debidamente aprovechado, al servicio de las necesidades de la sociedad. Tal vez el momento para la Salud Pública ha llegado. 